\title{
Global attractivity in a non-monotone age-structured model with age-dependent diffusion and death rates
}

\section{Al-Jararha ${ }^{1 *}$}

\section{"Correspondence:}

mohammad.ja@yu.edu.jo

'Department of Mathematics,

Yarmouk University, Irbid, Jordan

\section{Springer}

\begin{abstract} diffusive age-structured model

$$
\begin{cases}\frac{\partial u}{\partial t}+\frac{\partial u}{\partial a}=D(a) \frac{\partial^{2} u}{\partial x^{2}}-d(a) u, & t \geq t_{0} \geq A_{l}>0, a \geq 0,0<x<\pi \\ w(t, x)=\int_{\tau}^{A_{l}} u(t, a, x) d a, & t \geq t_{0} \geq A_{l}>0,0<x<\pi, \tau \geq 0, \\ u(t, 0, x)=f(w(t, x)), & t \geq t_{0} \geq A_{l}>0,0<x<\pi \\ u_{x}(t, a, 0)=u_{x}(t, a, \pi)=0, & t \geq t_{0} \geq A_{l}>0, a \geq 0\end{cases}
$$
\end{abstract}

In this paper, the global attractivity of the homogeneous equilibrium solution for the

is established when the diffusion and death rates, $D(a)$ and $d(a)$, respectively, are age dependent during the whole life of the species, and when the birth function $f(w)$ is nonmonotone. In the paper, we also present some demonstrative examples.

MSC: 34K10; 35K55; 35B35; 92D25

Keywords: Homogeneous equilibrium solution; Global attractivity; Age-structured model; Non-monotone birth function; Age-dependent death rate; Age-dependent diffusion rate

\section{Introduction}

Temporal maturation and spatial movement play a major role in many biological systems. Therefore, many mathematical models and studies have appeared recently dealing with the interaction between them; e.g., see [2-6, 8, 9, 11, 13-17, 20, 24, 26, 27, 29]. One of the most popular techniques that has been used to study the interaction between the temporal maturation and the spatial movement is the Smith-Thieme age-structure technique [17]. In this technique, the species population is divided into two categories: the immature population and the mature population. At different ages, the diffusive age-structured model is given by

$$
\frac{\partial}{\partial t} u(t, a, x)+\frac{\partial}{\partial a} u(t, a, x)=D(a) \frac{\partial^{2}}{\partial x^{2}} u(t, a, x)-d(a) u(t, a, x),
$$

where $u(t, a, x)$ is the size of the species population at any time $t \geq 0$, age $a \geq 0$, and a spatial location $x \in \Omega \subseteq \mathbb{R}$; the functions $D(a)$ and $d(a)$ are the age-dependent diffusion

(c) The Author(s) 2018. This article is distributed under the terms of the Creative Commons Attribution 4.0 International License (http://creativecommons.org/licenses/by/4.0/), which permits unrestricted use, distribution, and reproduction in any medium, provided you give appropriate credit to the original author(s) and the source, provide a link to the Creative Commons license, and indicate if changes were made. 
and death rates of the species; see [12]. Let $\tau \geq 0$ be the maturation time of the species and $A_{l}>0$ be its life span. Then the size of the mature population at any time $t \geq 0$ and a spatial location $x \in \Omega$ is given by

$$
w(t, x)=\int_{\tau}^{A_{l}} u(t, a, x) d a .
$$

Since only the mature individuals can reproduce, we assume that

$$
u(t, 0, x)=f(w(t, x))
$$

where $f(w)$ is a given birth function.

In [20], So et al. assumed that the diffusion and death rates of the mature population are age independent. Particularly, $D_{m}(a)=D$ and $d_{m}(a)=d$, where $D$ and $d$ are positive constants. By using this assumption and by applying the method of characteristics, So et al. derived the following reaction-diffusion equation:

$$
\frac{\partial}{\partial t} w(t, x)=D \frac{\partial^{2}}{\partial x^{2}} w(t, x)-d w(t, x)+u(t, \tau, x) .
$$

The function $u(t, \tau, x)$ appears above is called the maturation rate of the species, and is formulated by

$$
u(t, \tau, x)=\kappa \int_{-\infty}^{\infty} \Gamma_{\delta}(x-y) f(w(t-\tau, y)) d y,
$$

where

$$
\begin{aligned}
& \kappa=\exp \left[-\int_{0}^{\tau} d_{I}(a) d a\right], \\
& \delta=\int_{0}^{\tau} D_{I}(a) d a,
\end{aligned}
$$

and

$$
\Gamma_{\delta}(x)=\frac{\exp \left\{\frac{-x^{2}}{4 \delta}\right\}}{\sqrt{4 \pi \delta}} .
$$

The age-dependent functions $D_{I}(a)$ and $d_{I}(a)$ are the immature population diffusion and death rates, respectively. In conclusion, So et al. deduced the non-local time-delayed reaction-diffusion equation:

$$
\frac{\partial}{\partial t} w(t, x)=D \frac{\partial^{2}}{\partial x^{2}} w(t, x)-d w(t, x)+\kappa \int_{-\infty}^{\infty} f(w(t-\tau, y)) K_{\delta}(x-y) d y
$$

and they investigated the existence of monotone traveling wave solutions of this equation for the specific birth function $f(w)=p w \exp \left\{-a w^{\frac{1}{q}}\right\}$. In [11], Mei and So showed the stability of these traveling wave solutions. In [10], Liang and Wu investigated the existence of monotone traveling wave solutions of (1.5) for different birth functions. In [9], by using a numerical simulation, Liang et al. investigated the long time behavior of the solution 
of the age-structured model (1.1)-(1.3) when the spatial domain $\Omega$ is the closed interval $[0, l]$. In their study, Liang et al. assumed the diffusion and death rates of the mature population to be age independent, so that they can easily derive non-local time-delayed reaction-diffusion equations similar to equation (1.5).

In [24], Thieme and Zhao showed the existence of monotone traveling waves for the following stage-structured model:

$$
\begin{cases}\partial_{t} u(t, a, x)+\partial_{a} u(t, a, x)=D_{I}(a) \Delta_{x} u(t, a, x)-d_{I}(a) u(t, a, x), & 0<a<\tau, x \in \mathbb{R}^{n}, \\ u(t, 0, x)=f\left(u_{m}(t, x)\right), & t \geq-\tau, x \in \mathbb{R}^{n}, \\ \partial_{t} u_{m}(t, x)=D_{m} \Delta_{x} u_{m}(t, x)-d_{m} g\left(u_{m}(t, x)\right)+u(t, \tau, x), & t>0, x \in \mathbb{R}^{n},\end{cases}
$$

where $u(t, a, x)$ is the density of the species population at any time $t \geq-\tau$, age $a \geq 0$, and a location $x \in \mathbb{R}^{n}, u_{m}(t, x)$ is the density of the mature population at any time $t \geq-\tau$ and a location $x \in \mathbb{R}^{n}$, the functions $f\left(u_{m}\right)$ and $g\left(u_{m}\right)$ are the birth and mortality rates for the mature individuals, respectively, the age-dependent functions $D_{I}(a)$ and $d_{I}(a)$ are the diffusion and mortality rates for the immature population, respectively, and the positive constants $D_{m}$ and $d_{m}$ are the age-independent diffusion and death rates for the mature population, respectively. When the spatial domain is a closed and bounded set to $\mathbb{R}^{n}$, the above age-structured model has been investigated by Xu and Zhao [26] and by Jin and Zhao [29]. In these two studies, the authors transformed Eq. (1.6) to the following non-local time-delayed reaction-diffusion equation:

$$
\begin{cases}\partial_{t} u_{m}(t, x) & \\ \quad=D_{m} \Delta_{x} u_{m}(t, x)-d_{m} g\left(u_{m}(t, x)\right) & \\ \quad+\int_{\Omega} \Gamma(\chi(\tau), x, y) \mathcal{F}(\tau) f\left(u_{m}(t-\tau, y)\right) d y, & t>0, x \in \Omega, \\ B u_{m}(t, x)=0, & t \geq 0, x \in \partial \Omega, \\ u_{m}(t, x)=\psi(t, x), & t \in[-\tau, 0], x \in \Omega,\end{cases}
$$

where $\Gamma(\chi(\tau), x, y)$ is the Green's function associated with the Laplacian operator $\Delta_{x}$, $B u_{m}:=\frac{\partial u_{m}}{\partial n}+\beta u, \chi(\tau):=\int_{0}^{\tau} D_{I}(s) d s, \mathcal{F}(\tau):=e^{-\int_{0}^{\tau} d_{I}(s) d s}$, and $\psi(t, x)$ is a positive initial data. Particularly, Xu and Zhao in [26] investigated the global dynamics of (1.7) when the birth function $f\left(u_{m}\right)$ is monotonic; Jin and Zhao in [29] investigated the existence of asymptotic spreading speeds and the dynamics of periodic solutions.

Conclusively, all these studies assumed that the diffusion and death rates of the mature population to be age independent. In fact, many biological aspects could cause a variation in the diffusion and death rates among different ages of the mature individuals. For example, the reproductivity of the mature individuals varies among different ages. A particular example is the human population where women at the ages between 15 to 40 years have a lower death rate and a higher birth rate. Another example is the predation of animals where the predation of mature animals could be heavier on some certain age groups. Therefore, the death rate of the mature individuals varies dependently on the age of mature individuals. In epidemiology, the disease infection rate could be higher in some age groups of the mature populations. An example is the sexually transmitted diseases (STDs) that spread among the mature individuals, and it implies to a change in the death rate of the mature individuals depending on their age. Thus, it is more logistic to include the 
age effects of the mature population in the mathematical models. Therefore, Al-Jararha and $\mathrm{Ou}$ in [1] investigated the age-structured model (1.1)-(1.3) with the assumption that $D(a)$ and $d(a)$ are age-dependent functions during the whole life of the species. In their study, the authors considered two cases: the unbounded domains case and the bounded domains case. For the unbounded domains case, Al-Jararha and Ou derived from (1.1)(1.3) the following integral equation:

$$
w(t, x)=\int_{\tau}^{A_{l}} \int_{-\infty}^{\infty} b(w(t-s, y)) \frac{e^{-(x-y)^{2} / 4 \alpha(s)}}{\sqrt{4 \pi \alpha(s)}} \beta(s) d y d s, \quad t \geq A_{l},
$$

where

$$
\alpha(a)=\int_{0}^{a} D(\xi) d \xi
$$

and

$$
\beta(a)=\exp \left[-\int_{0}^{a} d(\xi) d \xi\right]
$$

and then they proved the existence of monotone traveling wave solutions for it. In the bounded domains case, particularly, $\Omega=[0, \pi]$, Al-Jararha and Ou derived from (1.1)(1.3) equipped with the Neumann boundary conditions the following integral equation:

$$
w(t, x)=\int_{\tau}^{A_{l}} \int_{0}^{\pi} \Pi(a, x, y) f(w(t-a, y)) d y d a, \quad t \geq A_{l},
$$

where the kernel function $\Pi(a, x, y)$ is given by

$$
\Pi(a, x, y)=\frac{\beta(a)}{\pi}\left(1+2 \sum_{n=1}^{\infty} e^{-n^{2} \alpha(a)} \cos n x \cos n y\right) .
$$

To study the dynamics of (1.9), particularly, the existence and the global stability of the homogeneous equilibrium solution of (1.9), Al-Jararha and $\mathrm{Ou}$ assumed that $f(w)$ is a monotone function. So, they can apply the theory of monotone dynamics and the comparison concepts to prove the desired result.

In this paper, we prove the global attractivity of such homogeneous equilibrium solution with the assumption that $f(w)$ is a non-monotone function. In this case, the theory of monotone dynamics and the comparison arguments cannot be applied. Therefore, to prove our main result, we apply the fluctuation method. The fluctuation method has been improved in [23] to study the global dynamics of the non-local time-delayed reactiondiffusion predator-prey model, and later it has been used in many mathematical articles to deal with the non-monotone dynamics difficulties; e.g., see [7, 18, 21-24, 31].

The paper is organized as follows. In Sect. 2, we present some preliminary results and concepts. In Sect. 3, we prove the main result. In Sect. 4, we present some demonstrative examples. Section 5 is devoted to the concluding remarks and discussions. 


\section{Preliminaries}

In this section, we present some preliminary concepts and some previous results. Let $\mathbb{X}=C([0, \pi], \mathbb{R})$ be the space of all continuous real valued functions defined on the closed interval $[0, \pi]$ equipped with the usual supremum norm:

$$
\|\psi\|_{\infty}=\sup _{x \in[0, \pi]}|\psi(x)|
$$

Let $\mathbb{X}^{+}=\{\psi(x) \in \mathbb{X} \mid \psi(x) \geq 0, x \in[0, \pi]\}$ be its positive cone. It is well known that $\operatorname{Int}\left(X^{+}\right) \neq \phi$. Hence, a strongly positive relation on $\mathbb{X}^{+}$can be defined. This relation is defined as follows: for any two functions $\eta$ and $\psi$ in $\mathbb{X}$, we have $\eta(x) \preceq \psi(x)$ if and only if $\eta(x) \leq \psi(x), \forall x \in[0, \pi]$. Thus, the pair $\left(\mathbb{X}, \mathbb{X}^{+}\right)$forms a strongly ordered Banach space. Also, we consider the functions space $\mathbb{Y}=C\left(\left[t_{0}-A_{l}, t_{0}\right], \mathbb{X}\right)$, where $t_{0} \geq A_{l}$ is fixed, with its ordered positive cone $\mathbb{Y}^{+}=C\left(\left[t_{0}-A_{l}, t_{0}\right], \mathbb{X}^{+}\right)$. For convenience, we identify each $\psi \in \mathbb{Y}^{+}$ as a function from $\left[t_{0}-A_{l}, t_{0}\right] \times[0, \pi]$ to $\mathbb{R}$ as follows: $\psi(s, x)=\psi(s)(x)$. For any function $\xi(\cdot):\left[t_{0}-A_{l}, c\right) \rightarrow \mathbb{X}$, where $c>t_{0}$, define $\xi_{t} \in \mathbb{Y}$ by $\xi_{t}(s)=\xi(t+s), \forall s \in\left[t_{0}-A_{l}, t_{0}\right)$. Let $M>0$. Then define the positive cone $\Sigma_{M}:=\left\{\psi(x) \in \mathbb{X}^{+} \mid \psi(x) \leq M, x \in[0, \pi]\right\}$ and the function space $\mathbb{Z}_{M}=C\left(\left[t_{0}-A_{l}, t_{0}\right], \Sigma_{M}\right)$. Assume that $f(w)$ is a Lipschitz continuous function. Then by applying the method of steps (for example, see [25]) the nonlinear integral equation

$$
\begin{cases}w(t, x)=\int_{\tau}^{A_{l}} \int_{0}^{\pi} \Pi(a, x, y) f(w(t-a, y)) d y d a, & t \geq A_{l}, x, y \in[0, \pi], \\ w_{x}(t, 0)=w_{x}(t, \pi)=0, & t \geq t_{0}, \\ w(s, x)=\psi(s, x) \geq 0, & t_{0}-A_{l} \leq s \leq t_{0}, x \in[0, \pi],\end{cases}
$$

where

$$
\Pi(a, x, y)=\frac{\beta(a)}{\pi}\left(1+2 \sum_{n=1}^{\infty} e^{-n^{2} \alpha(a)} \cos n x \cos n y\right),
$$

has a unique solution $w(t, x, \psi)$ for any $t \geq t_{0}$ and $\psi \in \mathbb{Y}^{+}$. Therefore, we can define the semiflow $\Phi(t): \mathbb{Y}^{+} \rightarrow \mathbb{Y}^{+}$, by $(\Phi(t) \psi)(s, x)=w(t+s, x, \psi), \forall s \in\left[t_{0}-A_{l}, t_{0}\right]$ and $x \in[0, \pi]$. In addition, the semiflow $\Phi(t): \mathbb{Y}^{+} \rightarrow \mathbb{Y}^{+}$is compact for $\forall t>t_{0}$; see [25]. The concept of the semiflow can be found in [30, p. 8] (also, one can see [19, p. 2]). We note that the kernel function $\Pi(a, x, y)$ is continuous, positive, and uniformly bounded on $\left[\tau, A_{l}\right] \times[0, \pi] \times$ $[0, \pi]$; see [1]. Moreover, $\int_{0}^{\pi} \Pi(a, x, y) d y=1$. So, if we set $\pi^{*}:=\int_{\tau}^{A_{l}} \int_{0}^{\pi} \Pi(a, x, y) d y d a$, then $\pi^{*}=\int_{\tau}^{A_{l}} \beta(a) d a$.

To prove our main result, we need the following assumptions on the birth function $f(w)$ :

(F) Assume that:

(F1) $f: \mathbb{R}^{+} \rightarrow \mathbb{R}$ is a Lipschitz continuous function $\forall w \geq 0, f(0)=0, f$ is a differentiable function at 0 with $f^{\prime}(0)=p>0$, and $f(w) \leq p w, \forall w \geq 0$.

(F2) There exists a positive constant $M$, such that $\forall w>M$ we have $\pi^{*} \bar{f}(w) \leq w$, where $\bar{f}(w):=\max _{v \in[0, w]} f(v)$. 
Consider the following linearized equation:

$$
\begin{cases}w(t, x)=p \int_{\tau}^{A_{l}} \int_{0}^{\pi} \Pi(a, x, y) w(t-a, y) d y d a, & t \geq A_{l}, x, y \in[0, \pi], \\ w_{x}(t, 0)=w_{x}(t, \pi)=0, & t \geq t_{0}, \\ w(s, x)=\psi(s, x) \geq 0, & t_{0}-A_{l} \leq s \leq t_{0}, x \in[0, \pi] .\end{cases}
$$

Let $w(t, x)=e^{\lambda t} w(x)$ in the above equation. Then we have the following eigenvalue problem:

$$
\left\{\begin{array}{l}
w(x)=p \int_{\tau}^{A_{l}} \int_{0}^{\pi} e^{-a \lambda} \Pi(a, x, y) w(y) d y d a, \quad x \in[0, \pi] \\
w_{x}(0)=w_{x}(\pi)=0
\end{array}\right.
$$

Set $w(x)=1$ in (2.4). Then the characteristic equation of (2.4) is given by $p \Gamma_{0}(\lambda)=1$, where $\Gamma_{0}(\lambda)=\int_{\tau}^{A_{l}} \exp \{-(\lambda a+\gamma(a))\} d a$ and $\gamma(a):=\int_{0}^{a} d(\xi) d \xi$. By solving this characteristic equation, we can uniquely determine the principal eigenvalue of (2.4). Clearly, $\Gamma_{0}(\lambda)$ is a decreasing function in $\lambda$ and it satisfies the following inequality:

$$
e^{(-\lambda \tau)} \int_{\tau}^{A_{l}} e^{-\gamma(a)} d a \leq \int_{\tau}^{A_{l}} \exp \{-(\lambda a+\gamma(a)) d a\} \leq e^{\left(-\lambda A_{l}\right)} \int_{\tau}^{A_{l}} e^{-\gamma(a)} d a .
$$

Thus, $\lim _{\lambda \rightarrow \infty} \Gamma_{0}(\lambda)=0, \lim _{\lambda \rightarrow-\infty} \Gamma_{0}(\lambda)=\infty$, and $\Gamma_{0}(0)=\pi^{*}:=\int_{\tau}^{A_{l}} e^{-\gamma(a)} d a>0$. Therefore, there exists a unique $\lambda_{0}$ that solves $p \Gamma_{0}(\lambda)=1$. So, $\lambda_{0}$ is the required principal eigenvalue of (2.4); see [1, Theorem 5.1]. Moreover, $\lambda_{0}>0$ if $p \pi^{*}>1$ and $\lambda_{0}<0$ if $p \pi^{*}<1$.

By following the same argument in the proof of Lemma 6.1 [1], we have the following theorem.

Theorem 2.1 Assume that (F1) and (F2) hold. Then the following statements are valid:

(I) for any $\psi \in \mathbb{Y}^{+}$, a unique solution $w(t, x, \psi)$ of (2.1) globally exists and $\lim \sup _{t \rightarrow \infty} w(t, x, \psi) \leq M$ uniformly for all $x \in[0, \pi]$.

(II) the semiflow $\Phi(t): \mathbb{Y}^{+} \rightarrow \mathbb{Y}^{+}$admits a connected global attractor on $\mathbb{Y}^{+}$which attracts every bounded set in $\mathbb{Y}^{+}$.

Also, by applying the same argument as in the proof of Lemma 6.2 and Theorem 6.3 in [1], we have the following theorem.

Theorem 2.2 Assume that (F1) and (F2) hold. Let $w(t, x, \psi)$ be a solution of (2.1) for $\psi \in$ $\mathbb{Y}^{+}$. Then the following statements hold:

(I) If $p \pi^{*}<1$ and $\psi \in \mathbb{Y}^{+}$, then $\lim _{t \rightarrow \infty} w(t, x, \psi)=0$.

(II) If $p \pi^{*}>1$, then (2.1) admits at least one positive homogeneous equilibrium solution $w^{*} \in[0, M]$, and there exists a positive constant $\sigma$ such that $\liminf _{t \rightarrow \infty} w(t, x, \psi) \geq \sigma$ uniformly, for all $\psi \in \mathbb{Y}^{+}$and $x \in[0, \pi]$.

Remark 2.1 Assume that $p \pi^{*}>1$ and assume that (F1) and (F2) hold. Let $F(w)=$ $\pi^{*} f(w)-w$. Since $f(w)$ satisfies (F1), $F(0)=0$ and $F^{\prime}(0)=p \pi^{*}-1>0$. Moreover, since $f(w)$ satisfies (F2), $F(M) \leq 0$. Therefore, there exists some $w^{*} \in(0, M]$ such that $F\left(w^{*}\right)=0$. Hence, $w^{*}$ is a positive homogeneous equilibrium solution of (2.1). 


\section{The main result}

In this section, we prove the global attractivity of the homogeneous equilibrium solution $w^{*}$. To prove this result we apply the fluctuation method. First, we start with the following definition.

Definition 3.1 The function $f(w):(0, M] \rightarrow \mathbb{R}$ satisfies the property (P); if for any $u, v \in$ $(0, M]$ with $u \leq w^{*} \leq v, u \geq \pi^{*} f(v)$, and $v \leq \pi^{*} f(u)$, then we have $v=u$.

Lemma 3.1 (Lemma 2.2 [7]) A function $f(w)$ satisfies the property (P) if one of the following statements hold:

(P0) $f(w)$ is a non-decreasing function on $[0, M]$.

(P1) $w f(w)$ is a strictly increasing function on $(0, M]$.

(P2) $f(w)$ is a non-increasing function for $w \in\left[w^{*}, M\right]$, and $\frac{f\left(\pi^{*} f(w)\right)}{w}$ is a strictly decreasing function for $w \in\left(0, w^{*}\right]$.

To prove our main result, we need more assumptions on the birth function $f(w)$; therefore we assume that

(F3) $f^{\prime}(0)>1, \frac{f(w)}{w}$ is a strictly decreasing function $\forall w \in(0, M]$, and $f(w)$ satisfies the property $(\mathrm{P})$.

Lemma 3.2 Let $\psi \in \mathbb{Y}^{+}$with $\psi\left(t_{0}, \cdot\right) \not \equiv 0$. Moreover, Let $\omega(\psi)$ be the omega limit set of the positive orbits through $\psi$ for the solution semiflow $\Psi(t)$. Then $\mathbb{Z}_{M}$ is positively invariant, i.e., $\Phi(t) \mathbb{Z}_{M} \subset \mathbb{Z}_{M}$. In addition, $\omega(\psi) \subset \mathbb{Z}_{M}$.

Proof Let $\psi \in \mathbb{Y}^{+}$with $\psi\left(t_{0}, \cdot\right) \not \equiv 0$, and let $\omega(\psi)$ be the omega limit set of the positive orbits through $\psi$ for the solution semiflow $\Phi(t)$. Then the conclusion of Theorem 2.1 implies that $\limsup _{t \rightarrow \infty} w(t, x, \psi) \leq M, \forall x \in[0, \pi]$. Hence, $\Phi(t) \mathbb{Z}_{M} \subset \mathbb{Z}_{M}$, and so, $\omega(\psi) \subset$ $\mathbb{Z}_{M}$.

Theorem 3.1 Assume that $p \pi^{*}>1$. Moreover, assume that (F1)-(F3) hold. Then, for any $\psi \in \mathbb{Y}^{+}$with $\psi\left(t_{0}, \cdot\right) \not \equiv 0$, we have $\lim _{t \rightarrow \infty} w(t, x, \psi)=w^{*}$ uniformly $\forall x \in[0, \pi]$.

Proof. To prove the global attractivity of $w^{*}$, by Lemma 3.2, it is sufficient to prove the global attractivity of $w^{*}$ on $\mathbb{Z}_{M}$. Therefore, let $\psi \in \mathbb{Z}_{M}$ be such that $\psi\left(t_{0}, \cdot\right) \not \equiv 0$. Then the solution of (2.1) through $\psi$ satisfies

$$
w(t, x)=\int_{\tau}^{A_{l}} \int_{0}^{\pi} \Pi(a, x, y) f(w(t-a, y)) d y d a .
$$

Let $w^{\infty}(x)=\lim \sup _{t \rightarrow \infty} w(t, x)$ and $w_{\infty}(x)=\liminf _{t \rightarrow \infty} w(t, x)$ for any $x \in[0, \pi]$. Then $w^{\infty}(x) \geq w_{\infty}(x)$. Since $p \pi^{*}>1$, by Theorem 2.2, we have

$$
0<\sigma \leq w_{\infty}(x) \leq w^{\infty}(x) \leq M
$$

Moreover, if we let $w^{\infty}=\sup _{x \in[0, \pi]} w^{\infty}(x)$ and $w_{\infty}=\inf _{x \in[0, \pi]} w_{\infty}(x)$, then $0<\sigma \leq w_{\infty} \leq$ $w^{\infty} \leq M$. Now, we define the diagonal function

$$
F(u, v)= \begin{cases}\min \{f(w): u \leq w \leq v\}, & \text { if } u \leq v \\ \max \{f(w): v \leq w \leq u\}, & \text { if } v \leq u\end{cases}
$$


Then $F(u, v):[0, M] \times[0, M] \rightarrow \mathbb{R}$ is a continuous function, non-decreasing in $u \in[0, M]$, non-increasing in $v \in[0, M]$, and $f(w)=F(w, w)$; see, e.g., [22, Sect. 3.6]. Since the kernel function $\Pi(a, x, y)$ is uniformly bounded for all $(a, x, y) \in\left[\tau, A_{l}\right] \times[0, \pi] \times[0, \pi]$, by Fatou's lemma, we have

$$
\begin{aligned}
w^{\infty}(x) & =\limsup _{t \rightarrow \infty} w(t, x) \\
& =\limsup _{t \rightarrow \infty} \int_{\tau}^{A_{l}} \int_{0}^{\pi} \Pi(a, x, y) f(w(t-a, y)) d y d a \\
& \leq \int_{\tau}^{A_{l}} \int_{0}^{\pi} \Pi(a, x, y) \limsup _{t \rightarrow \infty} f(w(t-a, y)) d y d a \\
& =\int_{\tau}^{A_{l}} \int_{0}^{\pi} \Pi(a, x, y) \limsup _{t \rightarrow \infty} F(w(t-a, y), w(t-a, y)) d y d a \\
& \leq \int_{\tau}^{A_{l}} \int_{0}^{\pi} \Pi(a, x, y) F\left(w^{\infty}, w_{\infty}\right) d y d a \\
& =\pi^{*} F\left(w^{\infty}, w_{\infty}\right) .
\end{aligned}
$$

Thus,

$$
w^{\infty}(x) \leq \pi^{*} F\left(w^{\infty}, w_{\infty}\right)
$$

Using the same argument, we have the following inequality:

$$
w_{\infty}(x) \geq \pi^{*} f\left(w_{\infty}, w^{\infty}\right)
$$

Obviously, by the definition of $F(u, v)$, there exist $u, v \in\left[w_{\infty}, w^{\infty}\right] \subset[0, M]$ such that $f(u)=$ $F\left(w^{\infty}, w_{\infty}\right)$ and $f(v)=F\left(w_{\infty}, w^{\infty}\right)$. Hence,

$$
f(u)=F\left(w^{\infty}, w_{\infty}\right) \geq \frac{w^{\infty}}{\pi^{*}} \geq \frac{u}{\pi^{*}} \quad\left(\frac{v}{\pi^{*}}\right)
$$

and

$$
f(v)=F\left(w_{\infty}, w^{\infty}\right) \leq \frac{w_{\infty}}{\pi^{*}} \leq \frac{v}{\pi^{*}} \quad\left(\frac{u}{\pi^{*}}\right)
$$

Consequently,

$$
\frac{\pi^{*} f(v)}{v} \leq 1=\frac{\pi^{*} f\left(w^{*}\right)}{w^{*}} \leq \frac{\pi^{*} f(u)}{u} .
$$

$\frac{f(w)}{w}$ is assumed to be a strictly decreasing function on $(0, M]$. Then $u \leq w^{*} \leq v$. Also, from (3.3) and (3.4), we get

$$
f(u) \geq \frac{w^{\infty}}{\pi^{*}} \geq \frac{v}{\pi^{*}}
$$

and

$$
f(v) \leq \frac{w_{\infty}}{\pi^{*}} \leq \frac{u}{\pi^{*}}
$$


That is,

$$
\pi^{*} f(u) \geq w^{\infty} \geq v \quad \text { and } \quad \pi^{*} f(v) \leq w_{\infty} \leq u
$$

Since $f(w)$ satisfies the property $(\mathrm{P}), w^{*}=u=v$. Moreover, we have

$$
\pi^{*} f(u) \geq w^{\infty} \geq u \text { and } f(v) \leq w_{\infty} \leq v .
$$

So, $w^{*}=w_{\infty}=w^{\infty}$. Recall that

$$
w^{\infty} \geq w^{\infty}(x) \geq w_{\infty}(x) \geq w_{\infty}, \quad \forall x \in[0, \pi] .
$$

Thus, $w^{\infty}(x)=w_{\infty}(x)=w^{*}, \forall x \in[0, \pi]$. Hence,

$$
\lim _{t \rightarrow \infty} w(t, x)=w^{*}, \quad \forall x \in[0, \pi] .
$$

To complete the proof, we need to show that $\lim _{t \rightarrow \infty} w(t, x)=w^{*}$ uniformly $\forall x \in[0, \pi]$. In fact, it is enough to show that $\omega(\psi)=\left\{w^{*}\right\}, \forall \psi \in \mathbb{Y}^{+}$. Let $\eta \in \omega(\psi)$. By the definition of the omega limit set, there exists a positive time sequence $t_{n} \rightarrow \infty$ such that $\Phi\left(t_{n}\right) \psi \rightarrow \eta$ in $\mathbb{Y}$ as $n \rightarrow \infty$. This implies that

$$
\lim _{n \rightarrow \infty} w\left(t_{n}+s, x, \psi\right)=\eta(s, x)
$$

uniformly for $(s, x) \in\left[t_{0}-A_{l}, t_{0}\right] \times[0, \pi]$. Hence, from (3.7), we have $\eta(s, x)=w^{*}, \forall(s, x) \in$ $\left[t_{0}-A_{l}, t_{0}\right] \times[0, \pi]$. It follows that $\omega(\psi)=w^{*}$. Thus $w(t, \cdot, \psi)$ converges to $w^{*}$ in $\mathbb{X}$ as $t \rightarrow \infty$.

\section{Examples}

In this section, we present some examples to demonstrate the applicability of the main result. First, we begin with the Nicholson blowflies birth function $f(w)=p w e^{-a w^{q}}$ where $a, p$, and $q$ are positive constants. Then we have the following theorem.

Theorem 4.1 Let $f(w)=p w e^{-a w^{q}}$, where $a>0, p>0$, and $q>0$. Assume that $1<\pi^{*} p \leq$ $e^{\frac{2}{q}}$. Then the unique positive steady state solution $w^{*}=\left[\frac{1}{a} \ln \left(p \pi^{*}\right)\right]^{\frac{1}{q}}$ attracts all positive solutions of (2.1).

Proof First, we remark that $f(w)$ satisfies the conditions (F1)-(F3), $f(w) / w$ is a strictly decreasing function on $[0, \infty)$. Moreover, $f^{\prime}(0)=p>0$, and $f(w)$ takes its maximum at the point $\bar{w}=\left(\frac{1}{a q}\right)^{\frac{1}{q}}$ and $f(\bar{w})=p\left(\frac{1}{a q e}\right)^{\frac{1}{q}}$. Assume that $1<\pi^{*} p \leq e^{\frac{1}{q}}$, then $f(w)$ is increasing function on $\left[0, w^{*}\right]$. Therefore, (P0) holds with $M=w^{*}$. Now, assume that $\pi^{*} p>e^{\frac{1}{q}}$. In this case, we consider $M=f(\bar{w})$. Hence, $f(w)$ is decreasing function on $\left[w^{*}, M\right]$. Moreover, the function

$$
h(w):=\frac{f\left(\pi^{*} f(w)\right)}{w}=p^{2} \pi^{*} \exp \left\{-a\left(w^{q}+\left(p \pi^{*} w\right)^{q} e^{-a q w^{q}}\right)\right\}
$$

is a strictly decreasing function on $\left[0, u^{*}\right]$ if $e^{\frac{1}{q}}<p \pi^{*} \leq e^{\frac{2}{q}}$. Thus, property (P2) holds, so the conditions of Theorem 3.1 are satisfied. As a result, $w^{*}$ attracts every positive solution of (2.1). 
Next, we consider the Beverton-Holt function $f(w)=\frac{p w}{1+a w^{q}}, a>0, p>0$, and $q>0$. Then we have the following theorem.

Theorem 4.2 Let $f(w)=\frac{p w}{1+a w^{q}}$, where $a>0, p>0$, and $q>0$. Assume that $q \in(0, \max (2$, $\left.\left.\frac{p \pi^{*}}{p \pi^{*}-1}\right)\right]$, or $q>\max \left(2, \frac{p \pi^{*}}{p \pi^{*}-1}\right)$ and $\pi^{*} f(\bar{w}) \leq\left(\frac{2}{a(q-2)}\right)^{\frac{1}{q}}$; where $p \pi^{*}>1$ and $\bar{w}$ is the value where $f(w)$ takes its maximum. Then the unique positive steady state solution $w^{*}=\left(\frac{p \pi^{*}-1}{a}\right)^{1 / q}$ attracts every positive solutions of (2.1).

Proof First, we remark that $f(w)$ satisfies the conditions (F1)-(F3), and $f(w) / w$ is a strictly decreasing function on $[0, \infty)$. Moreover, $f^{\prime}(0)=p>0$, and $f(w)$ takes its maximum at $\bar{w}=\left(\frac{1}{a(q-1)}\right)^{\frac{1}{q}}$ and $f(\bar{w})=\frac{p(q-1)}{q} \bar{w}$. Assume that $q \in(0,1]$, then $f(w)$ is monotone increasing on $[0, \infty)$, and hence, (P0) holds with $M=w^{*}$. Now, if we assume $1<q \leq 2$, then $w f(w)$ is increasing function on $[0, \infty)$. Hence (P1) holds with $M=w^{*}$. Moreover, if $1<p \pi^{*} \leq \frac{q}{q-1}$ (i.e., $\left.q \in\left(1, \frac{p \pi^{*}}{p \pi^{*}-1}\right)\right)$, then $w^{*} \leq \bar{w}$. Hence, if we let $M=w^{*}$, then (P0) holds. Conclusively, if $q \in\left(0, \max \left(2, \frac{p \pi^{*}}{p \pi^{*}-1}\right)\right]$, then either (P0) or (P1) holds. If $q>\max \left(2, \frac{p \pi^{*}}{p \pi^{*}-1}\right)$, then $h(w):=$ $w f(w)=\frac{p w^{2}}{1+a w^{q}}$ is a monotone increasing function on $\left[0,\left(\frac{2}{a(q-2)}\right)^{\frac{1}{q}}\right]$. Hence, if we consider $M=\pi^{*} f(\bar{w})$, then (P1) holds provided that $\pi^{*} f(\bar{w}) \leq\left(\frac{2}{a(q-2)}\right)^{\frac{1}{q}}$. Thus, the conditions of Theorem 3.1 hold, and so, $w^{*}$ attracts every positive solution of (2.1).

Finally, we consider the logistic function $f(w)=p w\left(1-\frac{w}{K}\right)$, where $p$ and $K$ are positive constants. Then we have the following theorem.

Theorem 4.3 Let $f(w)=p w\left(1-\frac{w}{K}\right), p>0$, and $K>0$ in (2.1). Moreover, assume that $1<p \pi^{*} \leq 3$. Then the unique positive steady state solution $w^{*}=K\left(1-\frac{1}{p \pi^{*}}\right)$ attracts every positive solution of (2.1).

Proof First, we remark that $f(w)$ satisfies the conditions (F1)-(F3), and $f(w) / w$ is a strictly decreasing function on $(0, K]$. Moreover, $f^{\prime}(0)=p>0$, and $f(w)$ takes its maximum at $\bar{w}=$ $\frac{K}{2}$ with $f(\bar{w})=\frac{p K}{4}$. Assume that $1<p \pi^{*} \leq 2$, then $f(w)$ is a monotone increasing function on $\left[0, \frac{2}{K}\right]$, and hence, (P0) holds with $M=u^{*}$. Assume that $2<p \pi^{*}<4$, and let $M=\frac{p \pi^{*}}{4} K$. Then the function

$$
h(w):=\frac{f\left(\pi^{*} f(w)\right)}{w}=\frac{p^{2} \pi^{*}}{K^{3}}\left(K^{2}(K-w)-p \pi^{*} w(K-w)^{2}\right)
$$

is a strictly decreasing function on $\left[0, w^{*}\right]$ provided that $2<p \pi^{*} \leq 3$. Hence, the property (P2) holds. Therefore, the assumptions of Theorem 3.1 hold. Thus $w^{*}$ attracts every positive solution of (2.1).

\section{Results and discussions}

Since many biological aspects could cause a variation in the diffusion and death rates among different ages of the mature individuals, it is important to investigate the dynamics of the ecological model (1.1)-(1.3) when the diffusion and death rates are age-dependent functions along the whole life of the species. For this purpose, the authors of [1] investigated (1.1)-(1.3) under this crucial assumption. In their paper they showed the existence of a unique positive and homogeneous equilibrium solution $w^{*}$, and they proved its global stability when the birth function $f(w)$ is monotone. If we assume that (F1)-(F2) hold, then 
(1.9) has a positive homogeneous equilibrium solution $w^{*}$. Moreover, if we assume that (F3) holds and the inequality $p \pi^{*}>1$ is satisfied, then $w^{*}$ is attracting every positive solution of (2.1). To show the implication of this result, we applied it to three types of birth functions.

We address a particular case; let $D(a)=D$ and $d(a)=d$, where $D$ and $d$ are positive constants, and let $f(w)=p w e^{-a w}$. Assume the life span of the species is large (i.e., $A_{l} \gg$ 1) and suppose that $1<\pi^{*} p \leq e^{2}$. Since $A_{l} \gg 1, \pi^{*} \sim \frac{e^{-d \tau}}{d}$. Thus, by Theorem 4.1, the positive equilibrium solution $w^{*}=\frac{1}{a} \ln \left(\frac{p e^{-d \tau}}{d}\right)$ is globally stable. Hence, by Theorem 2.2 and Theorem 4.1, the following two statements are valid:

(I) if $p e^{-d \tau}<d$, then the trivial solution is attracting every positive solution of (2.1);

(II) if $1<\frac{p e^{-d \tau}}{d} \leq e^{2}$, then the equilibrium solution $w^{*}=\frac{1}{a} \ln \left(\frac{p e^{-d \tau}}{d}\right)$ is attracting every positive solution of (2.1).

Here, we remark that a similar result to above threshold dynamics can be found in [28] when the diffusion and death rates are age independent.

In the paper, we employed the method of fluctuation to prove the global attractivity of a positive and homogeneous equilibrium solution $w^{*}$ of (1.9) in the case that $f(w)$ is a non-monotone function.

\section{Acknowledgements}

The author would like to thank the reviewers for their valuable comments on the paper. Also, the author would like to thank the deanship of graduate studies and scientific research at Yarmouk University for the partial support of this research.

\section{Funding}

Not applicable.

Availability of data and materials

Not applicable.

\section{Competing interests}

The author declares that he has no competing interests.

\section{Authors' contributions}

The author read and approved the final manuscript.

\section{Publisher's Note}

Springer Nature remains neutral with regard to jurisdictional claims in published maps and institutional affiliations.

Received: 4 June 2018 Accepted: 9 November 2018 Published online: 16 November 2018

\section{References}

1. Al-Jararha, M., Ou, C.: Populations dynamics with age-dependent diffusion and death rates. Eur. J. Appl. Math. 24, 471-500 (2013)

2. Diekmann, O.: Thresholds and traveling waves for the geographical spread of infection. J. Math. Biol. 6, 109-130 (1978)

3. Gourley, S.A., Kuang, Y.: Wavefronts and global stability in a time-delayed population with stage structure. R. Soc. Lond. Proc., Ser. A, Math. Phys. Eng. Sci. 459(2034), 1563-1579 (2003)

4. Gourley, S.A., So, J.W.-H.: Extinction and wavefront propagation in a reaction-diffusion model of a structured population with distributed maturation delay. Proc. R. Soc. Edinb. 133A, 527-548 (2003)

5. Gourley, S.A., So, J.W.-H., Wu, J.: Non-locality of reaction-diffusion equations induced by delay: biological modeling and nonlinear dynamics. J. Math. Sci. 124(4), 5119-5153 (2004)

6. Gourley, S.A., Wu, J.: Delayed non-local diffusive systems in biological invasion and disease spread. In: Nonlinear Dynamics and Evolution Equations. Fields Inst. Commun., vol. 48, pp. 137-200. Amer. Math. Soc., Providence, RI (2006). https://doi.org/10.1090/fic/048/06

7. Hsu, S.-B., Zhao, X.-Q.: Spreading speeds and traveling waves for nonmonotone integrodifference equations. SIAM J. Math. Anal. 40, 776-789 (2008)

8. Huddleston, J.V.: Population dynamics with age and time-dependent birth and death rates. Bull. Math. Biol. 45(5), $827-836(1983)$

9. Liang, D., So, J.W.-H., Zhang, F., Zou, X.: Population dynamics models with nonlocal delay on bounded domains and their numerical computation. Differ. Equ. Dyn. Syst. 11(1-2), 117-139 (2003) 
10. Liang, D., Wu, J.: Traveling waves and numerical approximations in a reaction advection diffusion equation with nonlocal delayed effects. J. Nonlinear Sci. 13, 289-310 (2003)

11. Mei, M., So, J.W.-H.: Stability of strong traveling waves for non-local time-delayed reaction diffusion equation. Proc. R. Soc. Edinb. 138A, 551-568 (2008)

12. Metz, J.A.J., Diekmann, O. (eds.): The Dynamics of Physiologically Structured Populations. Lecture Notes in Biomathematics, vol. 68. Springer, Berlin (1986). https://doi.org/10.1007/978-3-662-13159-6

13. $\mathrm{Ou}, \mathrm{C} ., \mathrm{Wu}, \mathrm{J}$.: Existence and uniqueness of a wavefront in a delayed hyperbolic-parabolic model. Nonlinear Anal. 63(3), 364-387 (2005)

14. Ou, C., Wu, J.: Persistence of wavefronts in delayed nonlocal reaction-diffusion equation. J. Differ. Equ. 235, 219-261 (2007)

15. Ou, C., Wu, J.: Traveling wavefronts in a delayed food-limited population model. SIAM J. Math. Anal. 39(1), 103-125 (2007)

16. Ou, G., Wu, J: Spatial spread of rabies revisited: influence of age-dependent diffusion on nonlinear dynamics. SIAM J. Appl. Math. 67(1), 138-163 (2006)

17. Smith, H., Thieme, H.: Strongly order preserving semiflows generated by functional differential equations. J. Differ. Equ. 93, 332-363 (1991)

18. Smith, H., Thieme, H.: Dynamical Systems and Population Persistence. Graduate Studies in Mathematics, vol. 118. Amer. Math. Soc., New York (2011)

19. Smith, H.L.: Monotone Dynamical Systems: An Introduction to the Theory of Competitive and Cooperative Systems. Math Surveys and Monographs, vol. 41. American Mathematical Society, Providence, RI (1995)

20. So, J.W.-H., Wu, J., Zou, X.: A reaction-diffusion model for a single species with age-structured. I. Traveling wavefronts on unbounded domains. Proc. R. Soc. Lond. A 457, 1841-1853 (2001). https://doi.org/10.1098/rspa.2001.0789

21. Thieme, H.R.: Persistence under relaxed point-dissipativity with applications to an endemic model. SIAM J. Math. Anal. 24(2), 407-435 (1993)

22. Thieme, H.R.: Density-dependent regulation of spatially distributed populations and their asymptotics speed of spread. J. Math. Biol. 195, 173-187 (2003)

23. Thieme, H.R., Zhao, X.-Q.: A non-local delayed and diffusive predator-prey model. Nonlinear Anal., Real World Appl. 2, $145-160(2001)$

24. Thieme, H.R., Zhao, X.-Q.: Asymptotic speeds of spread and traveling waves for integral equations and delayed reaction-diffusion model. J. Differ. Equ. 195, 430-470 (2003)

25. Wu, J.:Theory and Applications of Partial Functional Differential Equations. Applied Math. Sci., vol. 119. Springer, New York (1996)

26. Xu, D., Zhao, X.-Q.: A nonlocal reaction-diffusion population model with stage structure. Can. Appl. Math. Q. 11(3), 303-319 (2003)

27. Yi, T., Zou, X.: Global attractivity of the diffusive Nicholson blowflies equation with Neumann boundary conditions: a non-monotone case. J. Differ. Equ. 245, 3376-3388 (2008)

28. Yi, T., Zou, X.: Global dynamics of a delay equation with spatial non-locality in an unbounded domain. J. Differ. Equ. $251,2598-2611(2011)$

29. Yu, J., Zhao, X.-Q.: Spatial dynamics of a nonlocal periodic reaction-diffusion model with stage structure. SIAM J. Math. Anal. 40(6), 2496-2516 (2009)

30. Zhao, X.-Q.: Dynamical Systems in Population Biology. Springer, New York (2003)

31. Zhao, X.-Q.: Global attractivity in a class of nonmonotone reaction-diffusion equations with time delay. Can. Appl. Math. Q. 17(1), 271-281 (2009)

\section{Submit your manuscript to a SpringerOpen ${ }^{\circ}$ journal and benefit from:}

- Convenient online submission

- Rigorous peer review

- Open access: articles freely available online

- High visibility within the field

- Retaining the copyright to your article

Submit your next manuscript at $\gg$ springeropen.com 